\title{
Using Microbial Microcosms to Study Host-parasite Coevolution
}

\author{
Michael A. Brockhurst
}

Published online: 10 November 2009

(C) Springer Science + Business Media, LLC 2009

\begin{abstract}
Microbial microcosm experiments with bacteria and their viral parasites allow us to observe host-parasite coevolution in action. Laboratory populations of microbes evolve rapidly, thanks to their short generation times and huge population sizes. By taking advantage of a "living fossil record" stored in the laboratory freezer, we can directly compare the fitness of hosts and parasites with their actual evolutionary ancestors. Such experiments demonstrate that host-parasite coevolution is an important evolutionary force and a cause of strong and divergent natural selection.
\end{abstract}

Keywords Microcosm experiments .

Host-parasite coevolution · Experimental evolution

\section{Preamble}

In the exquisitely matched adaptations of intimately interacting species, Charles Darwin recognized the power of coevolution to shape the forms and behaviors of the organisms we see around us. He highlighted in the Origin of Species that the fit of a flower's nectar spur with the mouthparts of the pollinating insect could only have arisen through a series of coevolutionary adaptations and counteradaptations (Darwin 1859). However, although Darwin noted the life cycles of parasites as fine examples of adaptation, he may not have fully appreciated the potency of parasites as agents of natural selection or the power of

M. A. Brockhurst $(\bowtie)$

School of Biological Sciences, University of Liverpool,

Biosciences Building, Crown Street,

Liverpool L69 7ZB Merseyside, UK

e-mail: michael.brockhurst@liverpool.ac.uk host-parasite coevolution as a driver of evolutionary change (Schmid-Hempel 2009). It fell first to J.B.S. Haldane (Haldane 1992) and then to W.D. Hamilton (Hamilton 1980) to put host-parasite coevolution firmly into the spotlight. Its importance was neatly captured in the Red Queen Hypothesis (Van Valen 1973; Hamilton 1980). By this view, the struggle between hosts and parasites is ongoing because adaptation, increasing the fitness of one species, necessarily reduces the fitness of the other, resulting in continual reciprocal adaptations and counteradaptations just to maintain their fitness at its present level (Fig. 1). In the world of the Red Queen, hell is other species, and it is ecological interactions between these species that drive the bulk of evolutionary change.

One of the insights of Red Queen thinking was that host-parasite coevolution should drive very rapid evolution, so rapid in fact that biologists could expect to observe it occurring now. There is just one small problem with studying coevolution in nature: to really understand the nitty-gritty requires time travel! This is because the ideal experiment uses a "time-shift" such that host and parasite genotypes from the present day can be confronted with parasite or host genotypes from the past and future (Gandon et al. 2008; Gaba and Ebert 2009). Such time-shift experiments allow experimenters to make direct measurements of how both the infectivity of parasites and the resistance of hosts evolve through time. One solution to the time travel dilemma is to use laboratory populations of microbes which can be cryogenically stored in a freezer; these stored populations remain viable in suspended animation and form a "living fossil record" of evolutionary ancestors waiting to be defrosted at a later date (Buckling and Rainey 2002a; Brockhurst et al. 2003). A further advantage of using laboratory populations of microbes is their short generation times and large population sizes, which favor 


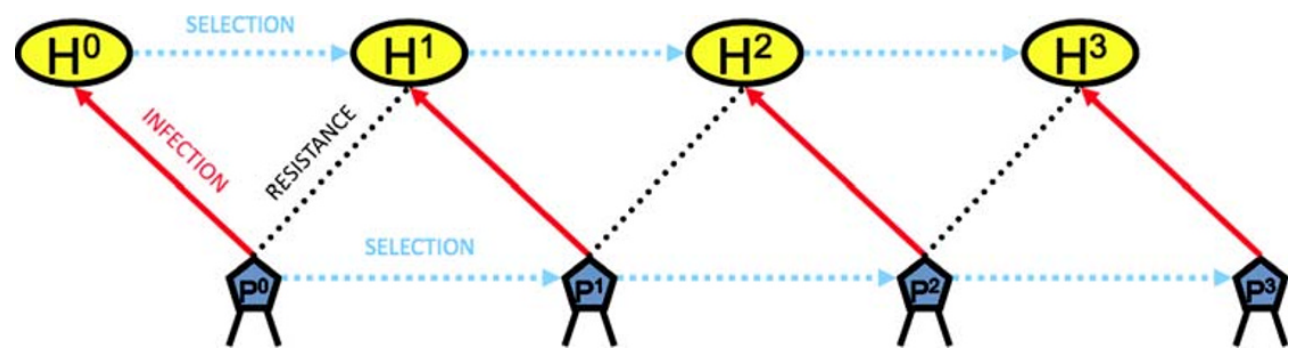

Fig. 1 A cartoon of host-parasite coevolution. $H$ denotes host, $P$ denotes parasite, solid red arrows denote successful infection, dotted black lines denote resistance, and dotted blue arrows denote natural selection for increased resistance or infectivity in hosts and parasites, respectively

very rapid evolution that is observable over the course of a few days (Elena and Lenski 2003; Jessup et al. 2004; Buckling et al. 2009). In this article, I discuss how evolutionary biologists have used laboratory populations of microbes to study host-parasite coevolution.

\section{Microcosms: The World in a Test Tube}

When viewed under an electron microscope, samples of seawater, soil, and freshwater are crammed with microbial life. In each milliliter, there may be upwards of one million bacterial cells, but ten times more numerous still are viruses, which are called bacteriophages or phages for short, that infect and kill bacteria (Weinbauer 2004). Morphologically, most phages look like lunar landing craft and consist of a head containing their genome, a cylindrical tail, and legs called tail fibers (Fig. 2a). Phages lead simple but deadly lives: they first attach to molecules on the surface of the bacterial cell using their tail fibers, next they inject their genome into the bacterium by contracting their tails like a hypodermic syringe; the phage genome then hijacks the machinery of the cell to make copies of itself, which finally burst out of the bacterium as fully formed phages ready to infect their next victim (Fig. 2b).

As you would expect in the world of the Red Queen, bacteria do not sit idly by while phages decimate them but, rather, have evolved an array of sophisticated defense mechanisms. These range from genetic mutations that change their surface molecules, thereby preventing phage attachment to internal mechanisms blocking phage replication and apoptotic cell-death postinfection to prevent further spread of the phages to neighboring cells (Allison and Klaenhammer 1998). Fortunately, it is easy to keep populations of bacteria and phages in the laboratory to study their coevolutionary arms-race in detail: all you need are test tubes containing liquid growth medium, an incubator, and a freezer to store the living fossil record. Each replicate population can be founded with genetically identical individuals (both bacteria and phages reproduce asexually, producing clones of themselves); but with millions of individuals per milliliter and genetic mutations arising from errors in the copying of DNA from generation to generation, natural selection soon has plenty to work
Fig. 2 Cartoons of a the anatomy of a phage and $\mathbf{b}$ the life cycle of a phage. Phage life cycle cartoon courtesy of Virginie Poullain

\section{B. Phage life-cycle}

A. Phage anatomy

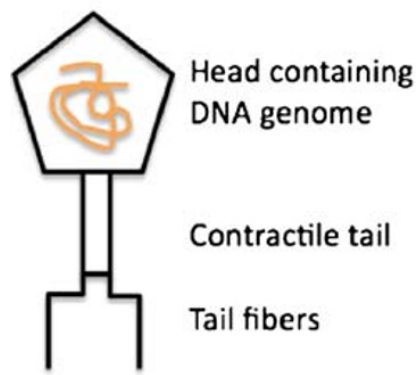

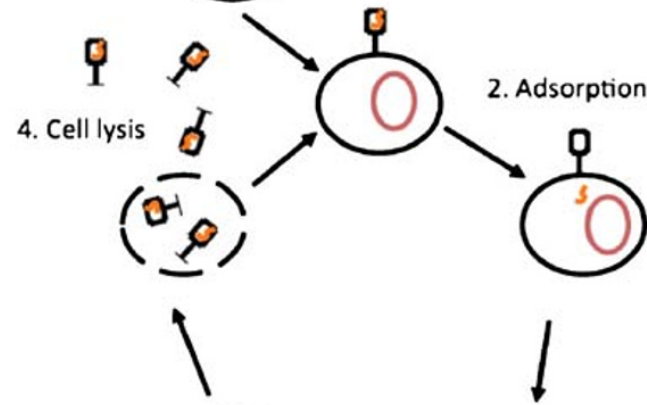

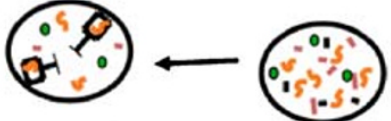

3. Phage replication 
with and evolutionary change in both species can be observed over the course of a few days!

\section{Fluctuation or Escalation?}

Two main models of host-parasite coevolution have been proposed, each corresponding to patterns seen in different host-parasite interactions in nature (Woolhouse et al. 2002). One proposes that host and parasite populations cycle through different resistance and infectivity genotypes through time, but that each genotype is a specialist only capable of resisting or infecting one genotype of the other species. The other proposes that new infectivity and resistance genotypes sweep through the population; repeated selective sweeps lead to the evolution of generalist hosts and parasites, which can resist or infect a progressively wider range of genotypes. The first model predicts genotype frequencies to fluctuate through time but for resistance and infectivity ranges to remain constant; whereas the second model predicts repeated fixation of new genotypes and escalation of resistance and infectivity ranges (Woolhouse et al. 2002).

We have investigated which type of coevolution occurs between bacteria and phage by coevolving replicate populations of the bacterium Pseudomonas fluorescens and phage $\Phi 2$ (pronounced phi-2) for several weeks in the laboratory, storing frozen samples of each population every two days (Brockhurst et al. 2003). We then performed time-shift experiments: at each sampled time step, we confronted bacteria from that time step with phages from two time steps previous, the same time step and two time steps in the future. We found that in nearly all cases, phages from the future were more infectious than present-day phages and that phages from the present day were more infectious than phages from the past against present-day bacteria (Fig. 3a). We next performed the experiment in reverse: holding the phages constant in time and testing them against hosts from the past, present, and future. Similarly, in the vast majority of cases, we found that bacteria from the future were more resistant than present-day bacteria and that bacteria from the present day were more resistant than bacteria from the past against present-day phages (Fig. 3b). Overall, these results strongly support the escalatory model of coevolution: as time goes by, bacteria and phages evolve to become more broadly resistant and more broadly infectious, respectively (Brockhurst et al. 2003). We have recently been able to tie down the genetic basis of this host-range expansion in the phages. This results from repeated selective sweeps of mutations that reduce the length of the phage tail-fiber proteins, presumably allowing the phages to attach deeper and deeper onto their target cell-surface molecules (Steve Paterson, personal communication).
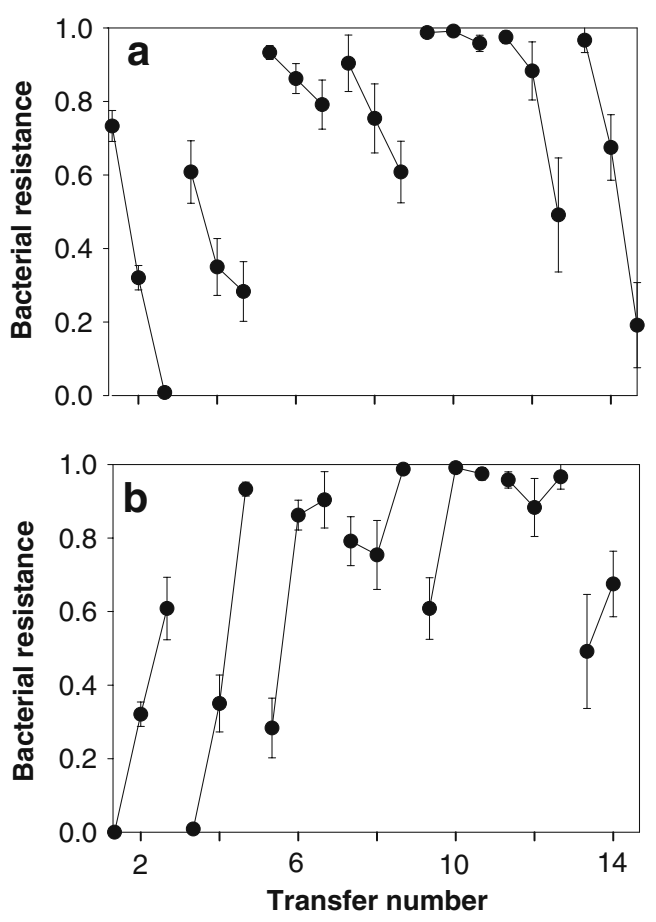

Fig. 3 Results of a time-shift experiment showing rates of a phage infectivity and $\mathbf{b}$ bacterial resistance evolution in coevolving populations of $P$. fluorescens and phage. In panel a lines represent bacterial resistance to past, contemporary, and future phage populations. In panel b lines represent resistance of past, contemporary, and future bacterial populations to a given phage population. The slope of each line provides a measure of the rate of evolutionary change over a four time step period. Adapted from Brockhurst et al. (2003)

\section{Coevolution in Space}

One of the most significant recent developments in our understanding of host-parasite coevolution in nature is the realization that coevolutionary interactions are often played out over large geographic scales. The importance of space in the coevolutionary process is captured in the Geographic Mosaic Theory (Thompson 2005). This comprises three components: (a) selection mosaics, which arise from each different population patch wandering off on its own coevolutionary trajectory; (b) coevolutionary hotspots and coldspots, such that the strength of selection arising from coevolution often varies between population patches; and (c) gene-flow, where dispersal of individuals can lead to mixing of the gene pools from different population patches. To test for differences in the trajectory of coevolution among populations, we need not a time-shift, but a "spaceshift" experiment where bacteria are confronted with phages from other populations at the same time step. If we do this with our coevolving laboratory populations, we see that bacteria are more resistant to phages from the same population than to phages from other populations (Buckling and Rainey 2002a; Morgan et al. 2005). This suggests that 
each population is indeed following its own slightly different coevolutionary trajectory, thereby creating selection mosaics.

It is surprisingly easy to alter the strength of coevolutionary selection to generate relative coevolutionary hotspots and coldspots in our laboratory populations. One way to do this is to increase the amount of nutrients in the growth medium, which effectively lowers the cost for bacteria of investing some of their resources in antiphage defenses (Lopez-Pascua and Buckling 2008). This change allows bacteria to more readily evolve resistance, which in turn imposes selection for novel infectivity genotypes in the phage, thus spurring on the rate of coevolution. Another way is to periodically shake the test tubes while they are being incubated. This has the effect of increasing the frequency with which bacteria and phage encounter one another; if bacteria encounter infectious phage more often, this increases natural selection for resistance, which in turn increases selection for novel infectivity in the phage, thereby accelerating coevolution (Brockhurst et al. 2003).

So what effect does dispersal (and therefore, gene flow) between different test tube populations have on coevolution between bacteria and phage? In general, dispersal between test tubes accelerates coevolution but only up to a point (Morgan et al. 2007; Vogwill et al. 2008). The reason for this relates to Fisher's Fundamental Theorem of Natural Selection: "The rate of increase in fitness of any organism at any time is equal to its genetic variance in fitness at that time" (Fisher 1930). Dispersal between populations on divergent coevolutionary trajectories increases the genetic diversity within populations upon which natural selection can act, thereby increasing the rate of evolution in both bacteria and phage (Morgan et al. 2007; Vogwill et al. 2008). However, dispersal is a double-edged sword because too much can homogenize genetic variation across all populations, reducing the benefits of dispersal, and causing the rate of coevolution to drop off at very high levels of dispersal (Vogwill et al. 2008). An interesting experimental twist is to allow moderate dispersal between relative coevolutionary hotspots and coldspots. When this occurs, we observe a pacemaker effect overriding local ecological conditions, such that dispersal from hotter spots to colder spots speeds up coevolution in the colder spot, whereas dispersal from colder spots to hotter spots slows down coevolution in the hotter spot (Vogwill et al. 2009).

\section{Escape and Radiate Coevolution}

Coevolution with parasites can also affect the evolution of other ecologically important traits in hosts. One interesting possibility is that evolutionary escape from parasite attack could open up a range of previously inaccessible ecological opportunities, thereby allowing diversification of the host so-called "escape and radiate coevolution" (Ehrlich and Raven 1964). In the absence of phage, P. fluorescens diversifies in laboratory test tubes into three types distinguishable by their heritable colony morphologies on agar plates and their niche preferences within the liquid medium (Rainey and Travisano 1998). Wrinkly spreaders live at the air-liquid interface, smooths live in the liquid broth, and fuzzy spreaders inhabit the murky depths at the bottom of the test tube. These types are each specialists in their preferred niche, and all three can stably coexist within the same population. When phages are added to populations of $P$. fluorescens, they prevent the diversification of the bacteria by reducing bacterial abundance and weakening resource competition (Buckling and Rainey 2002b). However, once bacteria evolve high levels of resistance, they are able to "escape and radiate" into the three colony types to take advantage of the ecological opportunities available in the test tube. The bacteria's success is short-lived, however, and phages rapidly evolve to overcome bacterial resistance, thereby reducing bacterial colony type diversity once more (Buckling and Hodgson 2007).

\section{Summary}

The coevolutionary interactions between bacteria and phage exhibit much of the complexities and nuances anticipated from theory. The hypotheses investigated experimentally in microcosms are of broad relevance to our understanding of host-parasite interactions in general, yet have proved difficult to test in natural populations. Microbial microcosms, therefore, provide an opportunity to observe coevolution in action and serve as an essential stepping stone between theory and the natural world.

Acknowledgments I am grateful to John Thompson and one anonymous reviewer for helpful comments on the manuscript.

\section{References}

Allison GE, Klaenhammer TR. Phage resistance mechanisms in lactic acid bacteria. Int Dairy J. 1998;8:207-26.

Brockhurst MA, Morgan AD, Rainey PB, Buckling A. Population mixing accelerates coevolution. Ecol Lett. 2003;6:975-9.

Buckling A, Hodgson DJ. Short-term rates of parasite evolution predict the evolution of host diversity. J Evol Biol. 2007;20:1682-8.

Buckling A, Rainey PB. Antagonistic coevolution between a bacterium and a bacteriophage. Proc R Soc Lond B. 2002a;269:931-6.

Buckling A, Rainey PB. The role of parasites in sympatric and allopatric host diversification. Nature. 2002b;420:496-9.

Buckling A, Maclean RC, Brockhurst MA, Colegrave N. The Beagle in a bottle. Nature. 2009;457:824-9. 
Darwin C. On the origin of species by means of natural selection, or the preservation of favoured races in the struggle for life. London: John Murray; 1859.

Ehrlich PR, Raven PH. Butterflies and plants - a study in coevolution. Evolution. 1964;18:586-608.

Elena SF, Lenski RE. Microbial genetics: evolution experiments with microorganisms: the dynamics and genetic bases of adaptation. Nat Rev Genet. 2003;4:457-69.

Fisher RA. The genetical theory of natural selection. Oxford: Clarendon; 1930.

Gaba S, Ebert D. Time-shift experiments as a tool to study antagonistic coevolution. Trends Ecol Evol. 2009;24:226-32.

Gandon S, Buckling A, Decaestecker E, Day T. Host-parasite coevolution and patterns of adaptation across time and space. $\mathrm{J}$ Evol Biol. 2008;21:1861-6.

Haldane JBS. Disease and evolution (Reprinted from La Ricerca Scientifica Supplemento, Vol 19, Pg 1-11, 1949). Curr Sci. 1992;63:599-604.

Hamilton WD. Sex versus non-sex versus parasite. OIKOS. 1980;35:282-90.

Jessup CM, et al. Big questions, small worlds: microbial model systems in ecology. Trends Ecol Evol. 2004;19:189-97.

Lopez-Pascua L, Buckling A. Increasing productivity accelerates host-parasite coevolution. J Evol Biol. 2008;21:853-60.
Morgan A, Gandon S, Buckling A. The effect of migration on local adaptation in a coevolving host-parasite system. Nature. 2005;437:253-6.

Morgan AD, Brockhurst MA, Lopez Pascua L, Pal C, Buckling A. Differential impact of simultaneous migration on coevolving hosts and parasites. BMC Evol Biol. 2007;7:1.

Rainey P, Travisano M. Adaptive radiation in a heterogeneous environment. Nature. 1998;394:69-72.

Schmid-Hempel P. Parasites - the new frontier: celebrating Darwin 2009. Biol Lett. 2009;5:625-7.

Thompson JN. The geographic mosaic of coevolution. Chicago: The University of Chicago Press; 2005.

Van Valen L. A new evolutionary law. Evol Theory. 1973;1:1-30.

Vogwill T, Fenton A, Brockhurst MA. The impact of parasite dispersal on antagonistic host-parasite coevolution. J Evol Biol. 2008;21:1252-8.

Vogwill T, Fenton A, Buckling A, Hochberg ME, Brockhurst MA. Source populations act as coevolutionary pacemakers in experimental selection mosaics containing hotspots and coldspots. Am Nat. 2009;173:e155-60.

Weinbauer MG. Ecology of prokaryotic viruses. FEMS Microbiol Rev. 2004;28:127-81.

Woolhouse ME, Webster JP, Domingo E, Charlesworth B, Levin BR. Biological and biomedical implications of the co-evolution of pathogens and their hosts. Nat Genet. 2002;32:569-77. 